\title{
Germanica
}

\section{Freundschaft. Zur Praxis eines Begriffs bei Frisch und Aristoteles}

Le concept d'amitié chez Frisch et Aristote

Friendship: Actualizations of a Concept in Frisch and Aristotle

\section{Olaf Berwald}

\section{OpenEdition}

Journals

Édition électronique

URL : http://journals.openedition.org/germanica/1204

DOI : 10.4000/germanica.1204

ISSN : 2107-0784

Éditeur

Université de Lille

Édition imprimée

Date de publication : 1 juillet 2011

Pagination : 131-139

ISBN : 9782913857278

ISSN : 0984-2632

\section{Référence électronique}

Olaf Berwald, «Freundschaft. Zur Praxis eines Begriffs bei Frisch und Aristoteles », Germanica [En ligne], 48 | 2011, document 8, mis en ligne le 01 juin 2013, consulté le 06 octobre 2020. URL : http:// journals.openedition.org/germanica/1204; DOI : https://doi.org/10.4000/germanica.1204

Ce document a été généré automatiquement le 6 octobre 2020.

(c) Tous droits réservés 


\title{
Freundschaft. Zur Praxis eines Begriffs bei Frisch und Aristoteles
}

\author{
Le concept d'amitié chez Frisch et Aristote \\ Friendship: Actualizations of a Concept in Frisch and Aristotle
}

\section{Olaf Berwald}

1 Sokratisch in seinem Mut, sich dem Wirklichen auszusetzen, ohne es unbesehen zu rigiden Nomenklaturen $\mathrm{zu}$ verfugen und rationaler Totalkontrolle verfügbar $\mathrm{zu}$ machen, betont Frisch in seinen 2010 posthum veröffentlichten Entwürfe[n] zu einem dritten Tagebuch die notwendige Prävenienz gelebter Erfahrung: "Ich bin auf Erfahrungen angewiesen, die mich begrifflich hilflos machen und von daher narrativ“" Keine in Aussagesätze gesperrten konzeptuellen Verortungsbemühungen werden einem lebendigen Sichaussetzen wandlungsfähiger Erfahrungen gewärtig, denn, so Frisch in seinem Tagebuch 1946-1949, "das Eigentliche, das Unsagbare, erscheint bestenfalls als Spannung zwischen diesen Aussagen“2.

2 Eine dieser grundlegenden, Abgründe öffnenden und in keinen Begriff und keine eindimensionale Aussage übersetzbaren Erfahrungen ist die Freundschaft. Die Unmöglichkeit und Unverzichtbarkeit von Freundschaft, ihr Scheitern und das gleichzeitige Beharren auf ihrer Lebbarkeit ist ein latenter und oft bestimmender thematischer Faden, mit dem Frischs Texte durchwirkt sind.

Eine diachrone Skizze, die Begriff und Praxis von Freundschaft in Max Frischs Werk und in der Nikomachischen Ethik des Aristoteles nachzeichnet, tut gut daran, sich in philologischer Bescheidenheit $\mathrm{zu}$ üben. Jeder Versuch, Korrelationen zwischen attischer philia und dem modernen Freundschaftsbegriff in Mittel- und Westeuropa zu erforschen, muss der Tatsache eingedenk bleiben, dass unser heutiges Verständnis von Individualität und somit auch von Freundschaft keine präzise Entsprechung in der Antike hat. Aber auch seit Frischs Werkepochen hat sich unsere Gebrauchsweise des Freund- und Selbstseins rasant verändert. Der von virtuellen sozialen Netzwerken propagierte Akkumulationszwang suggerierter Freundschaften verwischt zusehends die Konturen eines substantiellen Freundschaftsverständnisses. Mit weniger als 
sechshundert vermeintlichen Facebookfreunden kann man sich mit seinem digitalen Gesicht im Jahr 2011 kaum noch hervorwagen. Nicht nur zwischen Aristoteles und Frisch, sondern auch zwischen Frisch und uns haben sich semasiologische Risse im begrifflichen und praktischen Freundschaftsdiskurs aufgetan. Assoziationsfelder und konnotative Nuancen und Abschattungsskalen bleiben nicht konstant.

4 Auch die folgenden Ausführungen liefern kein stabiles konzeptuelles Gerüst. Sie laden zu künftigen Überschreibungen ein. Das Eingeständnis ihrer Vorläufigkeit ist der philologia, der freundschaftlichen Liebe zum verwundbaren Wort und seinen veränderlichen Sinnentfaltungen eingeschrieben.

In der Nikomachischen Ethik (EN) unterscheidet Aristoteles zwischen drei Arten von philia: Freundschaft um des gegenseitigen Nutzens willen, Freundschaft aus Lust und Freundschaft zwischen Guten (EN 1156a-1157a). In seinen Entwürfe[n] zu einem dritten Tagebuch (2010) gibt Frisch Beispiele für Freundschaftsphasen einseitiger Inanspruchnahme. Diese Momentaufnahmen von Freundschaftspraxis spitzen die aristotelische auf Nützlichkeit beruhende Freundschaftsvariante auf ein psychologisches Konsumverhältnis zu:

Unsere Gänge entlang der Seine: Gottfried Honegger, der meine Nöte sich anhört...

Unsere Gänge an der unteren Themse: Uwe Johnson, der meine Nöte sich anhört...

u.a.

Wie ich Freunde verbrauche!

Andere verbrauchen mich... ${ }^{3}$

6 Der parallele Satzbau indiziert die Austauschbarkeit von Orten und Freunden. Diverse landschaftliche Kulissen bilden den Hintergrund zu einer monotonen Dialogmechanik. Das narrative Ich in Frischs Tagebuchfragment gesteht sich selbst ein, dass es Freunde, unter ihnen den Maler und Bildhauer Gottfried Honegger und den Romancier Uwe Johnson, nicht nur wiederholt als verständnisvoll schweigende Zuhörer gebraucht, sondern auch als letztlich austauschbare Projektionsflächen verbraucht. Freunde als Rohstoffversorger behutsamen Zuhörens und diskreten Schweigens erhalten kein lebendiges Gesicht, sie werden von einem monologischen Ich zeitweise als vertrauliche Zuhörer vereinnahmt und $\mathrm{zu}$ einem passiv bleibenden Publikum und $\mathrm{zu}$ einer Oberfläche instrumentalisiert, die einen wirklichen Dialog lediglich schemenhaft andeutet.

7 Frischs Skepsis gegenüber einem Freundschaftsbegriff, der lediglich der Selbstberuhigung dient, geht so weit, dass er sich im Gedenken an den an Krebs gestorbenen Freund Peter Noll, den er in den Monaten vor seinem Tod fürsorglich begleitet und mit ihm sogar noch eine Reise nach Ägypten unternommen hatte, die (oder besser der) schonungslosen Frage nach der Möglichkeit freundschaftlicher Nähe und engen Vertrautseins stellt:

Wie befreundet bin ich mit Peter Noll? [...] der Bruder von Peter Noll war für zwei

Tage hier, wir redeten kaum über Peter, als scheuen sich beide zu verraten, wie

wenig sie ihn gekannt haben. [...] Wen habe ich wirklich gekannt? ${ }^{4}$

Es kann an dieser Stelle nicht darum gehen, biografischen Spekulationen über den Facettenreichtum von Frischs Freundschaften mit Friedrich Dürrenmatt, Peter Bichsel, Uwe Johnson, Otto F. Walter oder gar mit Ingeborg Bachmann nachzuhängen ${ }^{5}$. Der Konfliktreichtum der erotisch-hedonistischen Variante aristotelischer Freundschaftsmanifestationen in Frischs Text- und Lebensentwürfen kann in diesem Beitrag nicht ausgelotet werden. 
$9 \quad$ Entscheidend bleibt für diesen Essay die Frage nach den Strukturen und Eigenschaften von Freundschaft in Frischs Werk. Seine selbstkritische Skepsis gegenüber der Möglichkeit wirklicher, von energeia getragener Freundschaft impliziert, dass es Frisch stets um den Anspruch dessen geht, was in der Nikomachischen Ethik als die primäre und massgebliche Form der Freundschaft, der philia zwischen Guten diskutiert wird. Diese abstrakt anmutende Terminologie gewinnt an lebendiger Kontur, wenn wir ihr Frischs apostrophische Tagebuchnotiz an die amerikanische Freundin Alice Carey zur Seite stellen: "Du bist ein offener Mensch. [...] Du bist ein lauterer Mensch" ${ }^{\text {". }}$.

Inwiefern können die elegisch-panegyrischen Attribute Offenheit und Lauterkeit, die Frisch an dieser Stelle Alice Carey, dem lebendigen Vorbild der Lynnfigur in Montauk, zuschreibt, konkrete und einander verständliche Eigenschaften im Hier und Jetzt einer lebbaren Freundschaft werden? Eine Passage aus Frischs Tagebuch 1946-1949, die sich dem nach dem Zweiten Weltkrieg ebenso vermissten wie suspekt gewordenen Begriff Heimat widmet, kann uns ein Stück weiter helfen:

Heimat ist der Mensch, dessen Wesen wir vernehmen und erreichen. [...] nicht Einverständnis, das es nirgends so häufig gibt wie unter Wesensfremden, die einander mißdeuten, sondern Erreichbarkeit, und gerade wo man sich unter anderen Bedingungen trifft, erleben wir, durch keine gleichen Gewöhnungen getäuscht, das Verwandte oft um so reiner, um so überraschender und um so dankbarer, um so fruchtbarer ${ }^{7}$.

11 Die narzisstisch verzweifelte Konsenssucht und die Abhängigkeit von gekünstelter Harmonie wird in dieser Passage als das genaue Gegenteil von Freundschaft ausgemacht. Streitbare Unruhe statt statischem Einverständniskult gehört für Frisch zu einer lebendigen Freundschaft. Sie muss Widersprüche aushalten und darf sich nicht furchtsam vor ihnen abschotten wollen. Im spontanen gemeinsamen Lebendigsein, wie es Frisch in diesem Notat entwickelt, ermöglichen Erreichbarkeit, Vernehmbarkeit und wechselseitige spontane kommunikative Berührbarkeit Freundschaftserfahrungen, die von Dankbarkeit und Fruchtbarkeit geprägt sind. In Korrelation zu Aristoteles' Theorieentwurf von Freundschaft zwischen Guten entwirft Frisch ein Freundschaftsverständnis, das die Unabsehbarkeit und Unkontrollierbarkeit sich entfaltender Freundschaft als Bedingung gelingender, weil angstfreier Verbindung wahrnimmt.

In dialektischer Spannung zu Brechts Essay "Über das Anfertigen von Bildnissen“, in dem Brecht das den Anderen verbessernde Entgegenhalten eines neuen Ich- und Weltentwurfs als Freundschaftsdienst bezeichnet, hat für Frisch ein didaktisches und hierarchisches Element keinen Platz in der praktischen gegenseitigen Zuwendung von Freunden. Eine mit "Du sollst dir kein Bildnis machen" überschriebene Passage in Frischs Tagebuch 1946-1949 setzt mit dem Erstaunen über ein scheinbar paradoxes Phänomen ein:

Es ist bemerkenswert, daß wir gerade von dem Menschen, den wir lieben, am mindesten aussagen können, wie er sei. Wir lieben ihn einfach. Eben darin besteht ja die Liebe, das Wunderbare an der Liebe, daß sie uns in der Schwebe des Lebendigen hält, in der Bereitschaft, einem Menschen zu folgen in allen seinen möglichen Entfaltungen. Wir wissen, daß jeder Mensch, wenn man ihn liebt, sich wie verwandelt fühlt, wie entfaltet, und daß auch dem Liebenden sich alles entfaltet, das Nächste, das lange Bekannte. Vieles sieht er wie zum ersten Male. Die Liebe befreit es aus seinem Bildnis. Das ist das Erregende, das Abenteuerliche, das eigentlich Spannende, daß wir mit den Menschen, die wir lieben, nicht fertigwerden: weil wir sie lieben; solang wir sie lieben ${ }^{8}$. 
13 Freunde, so behauptet Frisch, bereichern uns um die Erfahrung des Erstaunens, des thaumazein, das laut Aristoteles den Beginn der Philosophie markiert. Die, die wir lieben, lassen sich nicht mit Begriffen und nicht in Worte fassen. Am befreundeten Menschen lieben wir die Entfaltbarkeit, das jeden Rahmen sprengende Überraschungspotenzial. Laut Frisch stellt Freundschaft ein ständiges und immer aufs Neue einsetzendes Befreiungsprojekt dar. Freundschaft ist lustvoll gewaltlose Bilderstürmerei.

Der Konditionalsatz am Ende des oben zitierten Fragments deutet die Gefährdung von Freundschaft an. Die Sucht nach Unveränderbarkeit und Ikonenhaftigkeit, die selbst noch dem geheuchelten Bedauern eines Mangels an Fluidität eignet, leitet stets das Ende einer Freundschaft ein. Frisch beschreibt die maskierte oder unverhohlene Einverleibungs- und Abstossungstendenz, die eine Freundschaft vergiftet, als einen Prozess, dessen Gewaltsamkeit und perspektivisch gebrochene Heuchelei er unterstreicht:

Man macht sich ein Bildnis. Das ist das Lieblose, der Verrat. [...] Wir sind es, die dem Freunde, dessen Erstarrtsein uns bemüht, im Wege stehen, und zwar dadurch, daß unsere Meinung, er sei erstarrt, ein weiteres Glied in jener Kette ist, die ihn fesselt und langsam erwürgt. Wir wünschen ihm, daß er sich wandle, o ja, wir wünschen es ganzen Völkern! Aber darum sind wir noch lange nicht bereit, unsere Vorstellung von ihnen aufzugeben. Wir selber sind die letzten, die sie verwandeln. Wir halten uns für den Spiegel und ahnen nur selten, wie sehr der andere seinerseits eben der Spiegel unseres erstarrten Menschenbildes ist, unser Erzeugnis, unser Opfer ${ }^{9}$.

In seiner ins Tagebuch 1946-1949 aufgenommenen Skizze "Der andorranische Jude“, die Frisch später zu seinem international wirkungsmächtigen Theaterstück Andorra (1961) ausarbeitete, diskutiert er die Tendenz, Menschen auf gerahmte Bilderrahmen zu spannen, mit einem Vergleich zu Religionsdiskursen:

Du sollst dir kein Bildnis machen, heißt es, von Gott. Es dürfte auch in diesem Sinne gelten: Gott als das Lebendige in jedem Menschen, das, was nicht erfaßbar ist. Es ist eine Versündigung, die wir, so wie sie an uns begangen wird, fast ohne Unterlaß wieder begehen - Ausgenommen wenn wir lieben ${ }^{10}$.

Die einzige Weise, der spiralförmigen Reziprozität wechselseitigen Abtötens durch Ikonisieren des Anderen (und seiner selbst) zu entgehen, ist nach Frisch die liebende Freundschaft, die das Gegenüber in keine Erwartungshorizonte einschmilzt.

Laut Frisch weist unser Verhalten Freunden gegenüber unvermutete Parallelen dazu auf, wie wir mit Gedanken umgehen. Sobald wir mit Menschen oder Gedanken wie mit Fertigprodukten hantieren, ist die Liebe zum Miteinandersein und die Lust am lebendigen Denken von Instrumentalisierungswut überschattet. Für Frisch dagegen ist Freundschaft begreifbar und erfahrbar als ethisch-epistemologische Behutsamkeit. Ein mit "Unterwegs" betitelter und somit Heideggers Essaysammlung Unterwegs zur Sprache (1950-59) in Titel und Inhalt vorwegnehmender Eintrag in Frischs Tagebuch 1946-1949 lautet:

Jeder Gedanke ist in dem Augenblick, wo wir ihn zum erstenmal haben, vollkommen wahr, gültig, den Bedingungen entsprechend, unter denen er entsteht; dann aber, indem wir nur das Ergebnis aussprechen, ohne die Summe seiner Bedingungen aussprechen zu können, hängt er plötzlich im Leeren, nichtssagend, und jetzt erst beginnt das Falsche, indem wir uns umsehen und Entsprechungen suchen [...] so stehen wir denn da und haben nichts als ein Ergebnis, erinnern uns, daß das Ergebnis vollkommen stimmte, beziehen es auf Erscheinungen, die diesen Gedanken selber nie ergeben hätten, überschreiten den Bereich seiner Gültigkeit, 
da wir die Summe seiner Bedingungen nicht mehr wissen, oder mindestens verschieben wir ihn - und schon ist der Irrtum da, die Vergewaltigung, die Überzeugung ${ }^{11}$. unabdinglich für perspektivische Luzidität sich selbst gegenüber bezeichnet wird, wird von Frischs Roman- wie Theaterfiguren nicht nachvollzogen. Anthony Price kommentiert Aristoteles wie folgt:

It is through observing the other, who is more directly visible to him than he is himself, that each discovers himself. And to maintain that discovery, even as he changes with age and develops with experience, each must maintain his friendships ${ }^{13}$. Elementarerkenntnis, indem sie die desaströsen Entwicklungen ihres Gegenteils durchspielen. Der Blindheit vortäuschende Gantenbein will niemanden entdecken, weder sich selbst noch seine (imaginierten) Mitmenschen. Frischs Figuren sind Isolationskünstler, denen ein Sichöffnenkönnen und somit ein Neuanfang nur punktuell und selbst dann nur illusorisch gegeben ist.

Stern-Gillet argumentiert, eine "primary, or virtue, friendship [...] constitutes a source of high self-realization for virtuous persons ${ }^{\text {"14 }}$. Es finden sich in Frischs Figurenarsenal kaum Spuren von sich selbst eine Wirklichkeit entwerfenden und Bestheit (arete) in Gemeinsamkeit anstrebenden Menschen. Damit bleibt auch die 
ausführlich von Nathalie von Siemens diskutierte aristotelische Trias von Freundschaft, Tätigkeit und Lust und das damit durchwirkte glückende Leben, die eudaimonia, ein telos, das Frischs Protagonisten nicht nur ständig verfehlen, sondern nur noch selten zu vermissen vermögen ${ }^{15}$.

In ihrer 2010 erschienenen Frischbiografie umschreibt Ingeborg Gleichauf Frischs programmatischen Anspruch an sich selbst, "Intelligenz der Mensch-Erfahrung" schreibend wach zu halten und auf "direkter Erfahrung von Menschsein" zu bestehen als Widerstand gegen ein bequemes und pseudorationales "Sich-Auskennen, Beurteilen aus dem Abstand heraus “ ${ }^{{ }^{16}}$. Gleichauf betont, dass Frischs Texte zu Leseakten anregen, die einer lebendigen Freundschaft gleichen. Die philia neugierig bleibender Lesepraxis eröffnet utopische Möglichkeiten:

In diesem Fall verbindet sich der utopische Ort Poesie mit dem utopischen Ort Freundschaft, und ein Zwiegespräch entsteht, mit dem Text und mit dem Menschen Frisch. (Gleichauf 254)

In ihrem 2011 erschienenen Buch Mein Name ist Frisch. Begegnungen mit dem Autor und seinem Werk betont Beatrice von Matt den experimentellen Charakter von Frischs unermüdlicher "Erkenntnisarbeit" und seinen "schonungslosen Willen zur Wahrheit [...] nicht zuletzt zur Wahrheit über sich selbst":

Er ist diese Aufgabe immer neu angegangen, in jeder der unterschiedlichen Phasen seines Werks, immer im Bemühen, sich und seiner jeweiligen Gegenwart gerecht zu werden ${ }^{17}$.

In Frischs Tagebuch 1946-1949 findet sich ein dringender Ausruf, aber eingeklammert, abgedämpft, und umgekehrt: Behutsam eingeklammert, aber mit der Dringlichkeit eines Ausrufezeichens versehen: "(Wirklich zu sein!) “. ${ }^{18}$ Nach Aristoteles umfasst eine tätige Wirklichkeit im Bestfall immer auch eine lebendige Freundschaft. Frisch schreibt der aristotelischen Praxis eine freundschaftsfähige energeia ein, die den befreundeten Menschen nie als kartographierbares epistemisches Objekt behandelt. Im inneren Dialog mit Goethe insistiert Frischs Werk auf der Wandlungsfähigkeit und Unabschliessbarkeit des Menschen.

Mit lakonischer Schärfe bekundet Frisch seine Mitarbeit am antiken und modernen Projekt des Zusammendenkens und Zusammenlebens von logos und mythos. Frischs Werk stellt ein sokratisches Experimentierfeld dar. Seine Texte verweigern sich der Nachfrage nach Lösungen. Sie laden nicht dazu ein, Menschen durchschauen zu wollen und bevorzugen stattdessen ein verwundbares offensein. $\mathrm{Zu}$ wahren und in fortwährenden sympoetischen Versuchen in Text und Leben immer wieder aufs Neue zu erfahren und zu bedenken bleibt nach Frisch das "Geheimnis, das der Mensch ja immerhin ist, ein erregendes Rätsel ${ }^{“ 19}$.

\section{NOTES}

1. Max Frisch, Entwürfe zu einem dritten Tagebuch. Hg. Peter von Matt. Berlin: Suhrkamp, 2010, S. 176. 
2. Max Frisch, Tagebuch 1946-1949. Frankfurt a.M.: Suhrkamp, 2007, S.37.

3. A.a.O., S. 61.

4. Max Frisch, Entwürfe zu einem dritten Tagebuch, a.a.O., S.114 u. 154.

5. Eine behutsame Behandlung von Frischs Freundschaften mit Dürrenmatt, Bichsel, Johnson, Walter und anderen findet sich bei Ingeborg Gleichauf, Jetzt nicht die Wut verlieren. Max Frisch eine Biografie. München: Nagel \& Kimche, 2010, S.244-53.

6. Max Frisch, Entwürfe zu einem dritten Tagebuch, a.a.O., S.127.

7. Max Frisch, Tagebuch 1946-1949, a.a.O., S.335.

8. Ebd., S. 27.

9. Ebd., S. 28-29.

10. Ebd., S.32.

11. Ebd., S.202.

12. Siehe hierzu Peter Schulz, Freundschaft und Selbstliebe bei Platon und Aristoteles. Freiburg/ München: Alber, 2000, S.303-312 sowie Nathalie von Siemens, Aristoteles über Freundschaft. Untersuchungen zur Nikomachischen Ethik VIII und IX. Freiburg/München: Alber, 2007, S.425.

13. Anthony W. Price, Love and Friendship in Plato and Aristotle. Oxford: Clarendon Press, 1989. Reprint 2004, S.124. Allerdings relativiert Price die Wichtigkeit dieser perspektivischen Argumentationslinie in , Anthony W. Price, "Friendship (VIII und LX)", in Die Nikomachische Ethik. Hg. Otfried Höffe, S.229-51. Berlin: Akademie Verlag, 1995. Hier: S.242-43, siehe hierzu auch Lorraine Smith Pangle, Aristotle and the Philosophy of Friendship. Cambridge University Press, 2003, S.187.

14. Stern-Gillet, Suzanne, Aristotle's Philosophy of Friendship. Albany: State University of New York Press, 1995, S.147.

15. Nathalie von Siemens, Aristoteles über Freundschaft. Untersuchungen zur Nikomachischen Ethik VIII und IX, a.a.O., S.357-403.

16. Gleichauf, Ingeborg, Jetzt nicht die Wut verlieren. Max Frisch - eine Biografie. München: Nagel \& Kimche, 2010, S.154. Gleichauf zitiert Frisch nach Forderungen des Tages. Porträts, Skizzen, Reden 1943-1982. Hg. Uwe Johnson. Frankfurt a.M.: Suhrkamp, 1983, S.93.

17. Beatrice von Matt, Mein Name ist Frisch. Begegnungen mit dem Autor und seinem Werk. München: Nagel \& Kimche, 2011, S.8.

18. Max Frisch, Tagebuch 1946-1949, a.a.O., S.203.

19. Ebd., S. 28.

\section{RÉSUMÉS}

La contribution d'Olaf Berwald examine la conception de l'amitié que Frisch développe surtout dans son Journal 1946-1949 et établit un lien philosophique avec la considération de la notion d'amitié que fait Aristote dans L'Éthique à Nicomaque. Un des principaux points de focalisation de l'essai de Berwald est la libération de la contrainte du préjugé, que Frisch pose comme exigence d'une amitié fondée sur la franchise.

Berwalds Beitrag untersucht das Verständnis von Freundschaft, das Frisch vor allem in seinem Tagebuch 1946-1949 entwickelt, und stellt es in einen symphilosophischen Zusammenhang mit der Diskussion des Freundschaftsbegriffs in Aristoteles' Nikomachischer Ethik. Ein Hauptaugenmerk 
von Berwalds Essay gilt der Befreiung vom Bebilderungszwang, die Frisch als Voraussetzung einer von Offenheit geprägten Freundschaft fordert.

Berwald's article examines the conception of friendship that Frisch develops primarily in his Sketchbook 1946-1949, and situates it in a symphilosophical nexus with the discussion of the concept of friendship in Aristotle's Nicomachean Ethics. The essay focuses on Frisch's claim that a friendship characterized by openness first and foremost has to liberate itself from the drive to cover the other with preconceived images.

INDEX

Mots-clés : amitié

oeuvrecitee Journal 1946-1949

\section{AUTEURS}

\section{OLAF BERWALD}

University of North Dakota 